\title{
FTO Gene: Association to Weight Regain after Lifestyle Intervention in Overweight Children
}

\author{
Thomas Reinehr $^{\mathrm{a}}$ Barbara Wolters $^{\mathrm{a}}$ Christian L. Roth ${ }^{\mathrm{c}}$ Anke Hinney $^{\mathrm{b}}$ \\ ${ }^{a}$ Department of Pediatric Endocrinology, Diabetes and Nutrition Medicine, Vestische Hospital for Children and \\ Adolescents Datteln, University of Witten/Herdecke, Datteln, and ${ }^{b}$ Department of Child and Adolescent Psychiatry, \\ Universitätsklinikum Essen, University of Duisburg-Essen, Essen, Germany; ${ }^{\mathrm{C} D e p a r t m e n t}$ of Pediatrics, Division of \\ Endocrinology and Diabetes, Center for Integrative Brain Research, Seattle Children's Research Institute, \\ University of Washington, Seattle, Wash., USA
}

\section{Key Words}

FTO - Obesity Children · Lifestyle intervention .

Weight regain

\begin{abstract}
Objective: Polymorphisms in intron 1 of the 'fat mass and obesity-associated' (FTO) gene are associated with weight status. We hypothesized that the risk allele at a polymorphism in intron 1 of FTO is associated with weight regain after end of lifestyle intervention. Methods: We longitudinally analyzed the changes of weight status as BMI-SDS in 346 unrelated overweight children (mean age $10.6 \pm 2.6$ years, $45 \%$ male, mean BMI-SDS $2.39 \pm 0.49$ ) both at the end of a 1-year lifestyle intervention and 1 year after the end of this intervention. We genotyped the obesity risk SNP rs9939609 at FTO by ARMS-PCR. Results: The children reduced their BMISDS $(-0.29 \pm 0.33 ; p<0.001)$ during intervention and increased their BMI-SDS between the end of intervention and 1 year later $(+0.10 \pm 0.41 ; p<0.001)$. The obesity risk allele at FTO SNP rs9939609 was not associated with BMI-SDS reduction during the lifestyle intervention $(p=0.622)$, but with weight regain 1 year after end of the intervention in multiple linear regression analyses adjusted for age, sex, pubertal stage, and baseline BMI-SDS (Bonferroni corrected $p=0.002$ ).
\end{abstract}

Conclusions: The obesity risk allele at a polymorphism in intron 1 of FTO was associated with weight regain 1 year after a 1-year lifestyle intervention.

(c) 2014 S. Karger AG, Basel

\section{Introduction}

Only a minor part of the high heritability of obesity has been unraveled thus far. Single nucleotide polymorphisms (SNP) in intron 1 of 'fat mass and obesity-associated' (FTO) confer the most relevant polygenic effect on body weight [1]. Associations of these polymorphisms with BMI were reported in several studies in both children and adults [2-4]. However, some studies did not confirm this finding $[5,6]$.

Association between the obesity risk alleles and weight loss in lifestyle interventions was equivocal [710]. Some studies did not find a relationship between FTO polymorphism(s) and weight loss [8-10] in contrast to others $[7,11]$. The reasons for these different findings are unclear. While the role of dietary intake in

This study is registered at clinicaltrials.gov (NCT00435734).

\section{KARGER}

E-Mail karger@karger.com

www.karger.com/hrp (c) 2014 S. Karger AG, Basel

$1663-2818 / 14 / 0816-0391 \$ 39.50 / 0$
Prof. Dr. Thomas Reinehr

Department of Pediatric Endocrinology, Diabetes and Nutrition Medicine Vestische Hospital for Children and Adolescents Datteln, University of Witten/Herdecke Dr. F. Steiner Strasse 5, DE-45711 Datteln (Germany)

E-Mail T.Reinehr@kinderklinik-datteln.de 
the relationship between FTO polymorphism(s) and weight status is controversially being discussed $[5,12]$, a significant relationship of physical exercise on the association between FTO polymorphism(s) and weight status have been reported: lifestyle interventions with intensive physical exercise did not find a relationship between FTO polymorphism(s) and weight loss [8-10] in contrast to interventions without intensive physical exercise units $[7,11]$. Therefore, the hypothesis has been generated that intensive physical exercise may overcome the effect of FTO polymorphism(s) on weight status $[3,4]$.

Due to these conflicting findings and the lack of studies in children analyzing the impact of SNPs in FTO to weight regain after weight loss, we performed the following study in obese children to analyze whether a SNP in FTO is associated with weight regain after end of intervention when regular physical exercise was stopped. Studies in obese children seem preferable since genetic causes of obesity likely show impact in childhood. Furthermore, studies in children have the advantage that there is no potential confusion with other diseases, medications, or active tobacco smoking. We hypothesize that a FTO polymorphism is associated with weight regain after lifestyle. Furthermore, we analyzed descriptively the relationships between a FTO polymorphism and cardiovascular risk factors at baseline as well as the association of the alleles at this polymorphism to changes of the cardiovascular risk factors.

\section{Methods}

The local ethics committees of the Universities of Witten/Herdecke and Duisburg-Essen approved this study. Written informed consent was obtained from all subjects and their parents.

\section{Subjects}

The investigated overweight children presented consecutively to our outpatient center specialized in pediatric obesity. All individuals were of European descent. Inclusion criteria were overweight according to the definition of International Task Force of childhood obesity [13] and participation in the 1-year lifestyle intervention 'Obeldicks' from 2008 to 2011 with an available followup measurement 1 year after the end of the intervention. Exclusion criteria were any medications or any endocrine or syndromal disorder.

\section{Measurements}

Weight status was determined as BMI at baseline, at the end of the 1-year intervention, and again 1 year later (i.e. 2 years after baseline). Height was measured to the nearest millimeter. Weight was measured in underwear to the nearest $0.1 \mathrm{~kg}$ using a cali- brated balance scale. The degree of overweight was quantified using Cole's least mean square method, which normalized the BMI skewed distribution and expressed BMI as a standard deviation score (BMI-SDS) [14]. The pubertal developmental stage was determined according to Marshall and Tanner, and categorized into three groups based on breast and genital stages (prepubertal: boys with genital stage I, girls with breast stage I; midpubertal: boys with genital stage II-III, girls with breast stage IIIII; late/post-pubertal: boys with genital stage $\geq I V$, girls with breast stage $\geq \mathrm{IV}$ ).

At baseline and at the end of intervention, cardiovascular risk factors (waist circumference, blood pressure, fasting lipids, glucose, and insulin) were determined. Waist circumference was measured halfway between lower rib and iliac crest. Blood pressure was measured using a validated protocol [15]. Blood pressure was measured at the right arm after a 10-min rest in the supine position with an oscillometric device (Omron M6). Two repeated recordings were made with $5 \mathrm{~min}$ in between and the lowest value of the two recordings of systolic blood pressure and diastolic blood pressure measurements was recorded. The cuff size was based on the length and circumference of the upper arm and was as large as possible without having the elbow skin crease obstructing the stethoscope [15].

Blood sampling was performed at 8 a.m. after $10 \mathrm{~h}$ fasting. After clotting, blood samples were centrifuged for $10 \mathrm{~min}$ at 8,000 $\mathrm{rpm}$. Serum was stored at $-81^{\circ} \mathrm{C}$ for later determination of insulin. Insulin concentrations were measured by microparticle-enhanced immunometric assay (MEIA ${ }^{\mathrm{TM}}$, Abbott, Wiesbaden, Germany). Glucose levels were determined by colorimetric test using a Vitros ${ }^{\mathrm{TM}}$ analyzer (Ortho Clinical Diagnostics, Neckargemünd, Germany). Triglyceride, LDL and HDL cholesterol, and glucose concentrations were measured using commercially available test kits (HDL-C- Plus ${ }^{\mathrm{TM}}$ and LDL-C-Plus ${ }^{\mathrm{TM}}$, both Roche Diagnostics, Mannheim, Germany; Vitros ${ }^{\mathrm{TM}}$ analyzer, Ortho Clinical Diagnostics; MEIA ${ }^{\mathrm{TM}}$, Abbott). Homeostasis model assessment (HOMA) was used to detect the degree of insulin resistance using the formula: Resistance $(\mathrm{HOMA})=($ insulin $[\mathrm{mU} / \mathrm{l}] \times$ glucose $[\mathrm{mmol} / 1]) / 22.5[16]$.

\section{Genotyping}

SNP genotyping for rs9939609 (intron 1 of FTO) was performed by allele-specific polymerase chain reaction (ARMS-PCR) as reported previously [10]. For validity of genotypes, alleles were rated independently by at least two experienced individuals. Discrepancies were resolved unambiguously either by reaching consensus or by retyping.

\section{Intervention}

The 1-year lifestyle intervention 'Obeldicks' has been described in detail elsewhere [17]. Briefly, this intervention is based on intensive physical exercise ( $1.5 \mathrm{~h} /$ week), nutrition education, and behavior therapy including the individual psychological care of the child and its family. The exercise therapy consists of sports, instructions in physical exercise as part of daily life, and in reduction of the amount of time spent watching television. The nutritional course is based on the prevention concept of the 'optimized mixed diet' which is both fat- and sugar-reduced. After the end of the intervention, no regular physical exercise was offered any more at the treatment center. The intervention was offered at three treatment centers in Germany. 


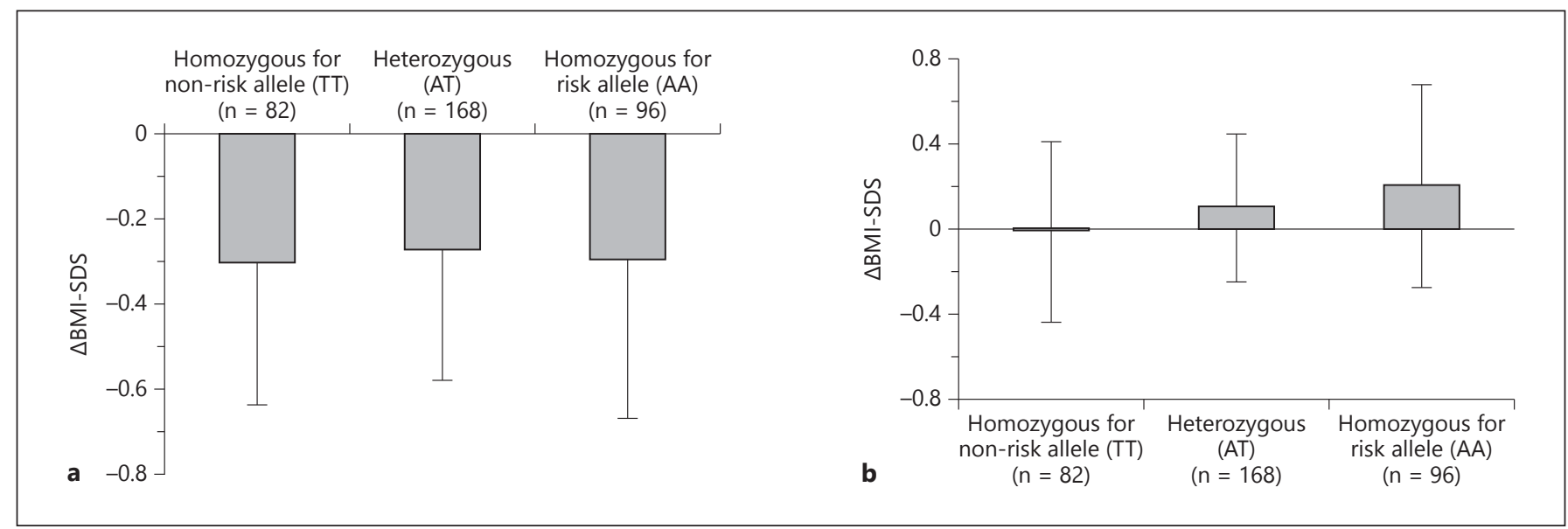

Fig. 1. Changes of BMI-SDS during a 1-year lifestyle intervention (a) and during a 1-year follow-up period after the end of the intervention (b) in 346 overweight children separated to polymorphism

\section{Statistics}

Statistical analyses were performed using Winstat ${ }^{\mathrm{TM}}$ for Excel. We used standard methods for descriptive statistics (such as means \pm SD). Changes of BMI-SDS were compared by paired Student's tests. We used an additive genetic model for the impact of the FTO SNP on BMI-SDS change or on cardiovascular risk factors or their changes during lifestyle intervention. Multiple linear regression analyses with BMI-SDS changes, BMI changes, cardiovascular risk factors, or changes of cardiovascular risk factors as dependent variables, and age, sex, baseline pubertal stage, changes of pubertal stage, baseline BMI-SDS, treatment center, and the FTO SNP as independent variables were calculated. Treatment center, pubertal stage, and change of pubertal stage were used as categorical variables in these models. All reported $\mathrm{p}$ values are two-sided and nominal. Corrections for multiple testing were performed by Bonferroni adjustment. The associations between cardiovascular risk factors and the FTO SNP were performed exploratively.

\section{Results}

A total of 346 unrelated overweight children (mean age $10.6 \pm 2.6$ years, $45 \%$ male, mean BMI $27.3 \pm 4.2$, mean BMI-SDS $2.39 \pm 0.49)$ were included in this analysis. $82(23.7 \%)$ children were homozygous for the obesity non-risk allele (TT), 168 (48.6\%) heterozygous (AT), and $96(27.7 \%)$ homozygous for the obesity risk allele (AA). The genotype distribution did not depart from the Hardy-Weinberg equilibrium.

The 346 overweight children reduced their weight significantly $(\Delta B M I-S D S-0.29 \pm 0.33 ; \mathrm{p}<0.001)$ during the 1 -year lifestyle intervention. The children increased their rs9939609 at FTO (data as mean and SD, negative values for the changes $(\Delta)$ of BMI-SDS indicate a reduction of BMI-SDS, $A$ is the risk allele at $F T O)$.

Table 1. Effect of the obesity risk allele (A) at the rs9939609 polymorphism in intron 1 of FTO on body weight loss (as $\triangle$ BMI-SDS) during and after a 1-year lifestyle intervention in 346 overweight children

\begin{tabular}{llll}
\hline & $\begin{array}{l}\Delta \text { BMI-SDS } \\
\text { during } \\
\text { 1 year } \\
\text { intervention }\end{array}$ & $\begin{array}{l}\Delta \text { BMI-SDS } \\
\text { between end of } \\
\text { intervention and } \\
1 \text { year later }\end{array}$ & $\begin{array}{l}\Delta \text { BMI-SDS } \\
\text { between } \\
\text { baseline at } \\
\text { 2 years later }\end{array}$ \\
\hline $\begin{array}{llll}\Delta \text { BMI-SDS, } \beta \text {-estimator } \\
\text { 95\% CI }\end{array}$ & -0.01 & -0.09 & -0.08 \\
$\mathrm{r}^{2}$ & $-0.06,0.04$ & $-0.14,-0.04$ & $-0.15,0.01$ \\
$\mathrm{p}$ value & 0.06 & 0.04 & 0.02 \\
\hline
\end{tabular}

The $\beta$ estimators were derived from linear regression models: additive model of inheritance, adjustment for age (linear), sex, pubertal stage at baseline, change of pubertal stage, treatment center, and baseline BMI-SDS. Positive values of the effect sizes for the changes $(\Delta)$ indicate a reduction of BMI-SDS. Results that were significant after correction for multiple testing are highlighted in bold. * Adjusted $\mathrm{p}$ value.

BMI-SDS significantly between end of intervention and 1 year later $(+0.10 \pm 0.41$ BMI-SDS; $\mathrm{p}<0.001$, adjusted $\mathrm{p}<0.001)$. Compared to baseline, BMI-SDS at the 2 -year follow-up was still significantly reduced $(-0.18 \pm 0.46$; $\mathrm{p}<$ 0.001 , adjusted $\mathrm{p}<0.001$ ).

The studied obesity risk allele at the SNP near FTO was not associated with BMI-SDS reduction during the lifestyle intervention but with weight regain 1 year after the end of the intervention (table 1; fig. 1). 
Table 2. Cardiovascular risk factors and their changes during the 1-year lifestyle intervention separated to the rs 9939609 genotype at FTO

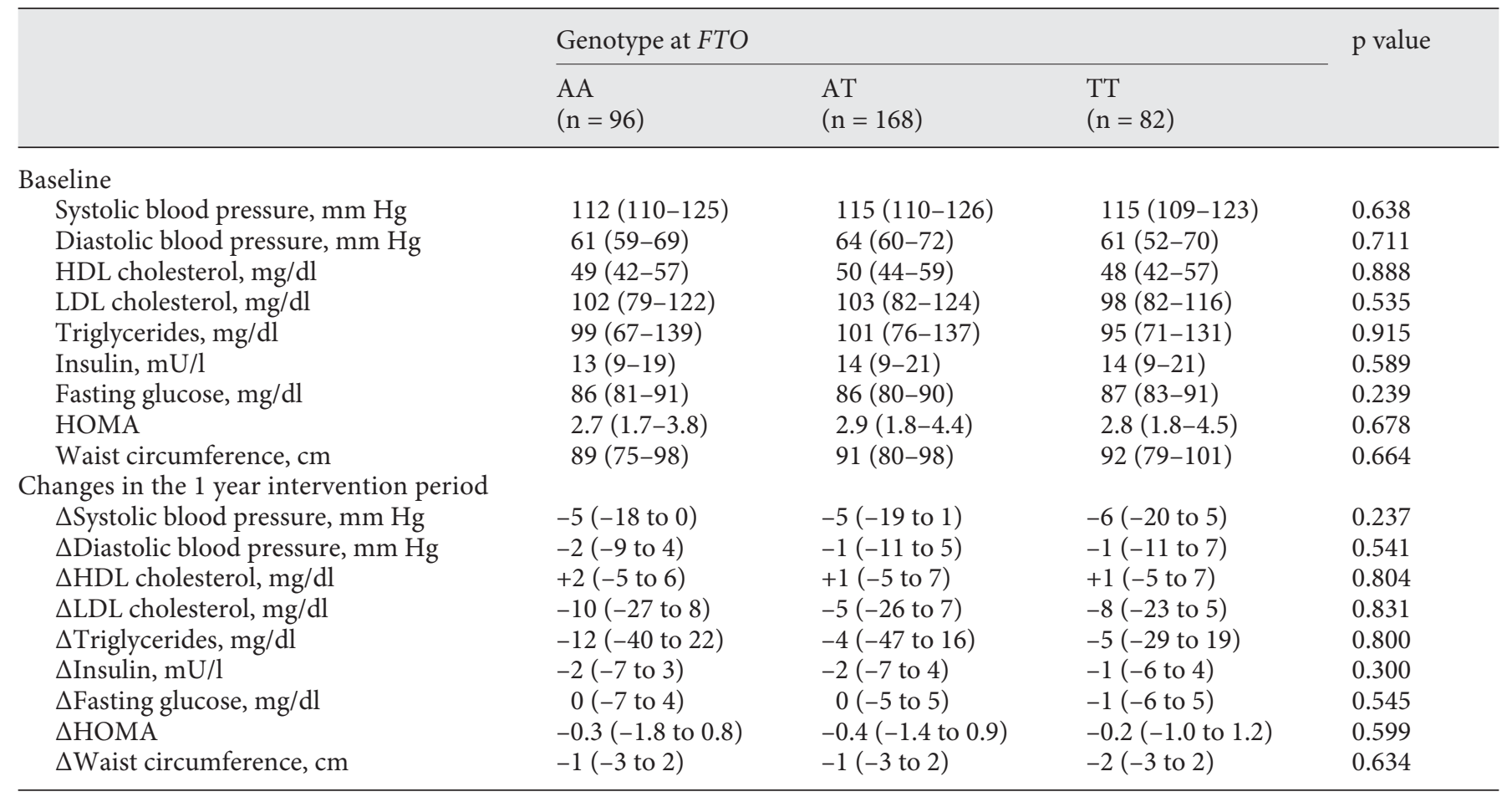

p value factors derived from multiple linear regression analyses: additive model of inheritance, adjustment for age (linear), sex, pubertal stage at baseline, and baseline BMI-SDS. Positive values of the effect sizes for the changes $(\Delta)$ indicate an increase. Data are median and interquartile range.

Summing the time periods of intervention and followup demonstrated that the SNP near FTO was not associated significantly with changes of BMI-SDS between baseline and 2 years later after correcting for multiple testing.

Using changes of BMI instead the changes of BMI-SDS revealed the same findings: the A allele at the SNP near FTO was not associated with BMI reduction during the lifestyle intervention in multiple linear regression analysis adjusted to age, pubertal stage, gender, and baseline BMI $(\mathrm{p}=0.777)$. The risk allele $(\mathrm{A})$ was significantly related to BMI increase after the end of lifestyle intervention in multiple linear regression analysis $\left(\mathrm{r}^{2}=0.08\right)$ adjusted to age, pubertal stage, gender, and baseline BMI ( $\beta$-coefficient $0.71,95 \%$ confidence interval $0.04-1.38$, $\mathrm{p}=0.039)$.

None of the cardiovascular risk factors at baseline or their changes during the lifestyle intervention were significantly associated to the rs 9939609 obesity risk alleles at FTO (table 2).

\section{Discussion}

We demonstrated that the obesity risk allele (A) at the FTO SNP rs9939609 was associated with higher weight regain after completion of a lifestyle intervention program. However, as this effect was small, other factors are likely involved as well [18].

On the other side, this SNP at the FTO was not associated to weight loss during the lifestyle intervention in concordance to previous larger studies in adults [8] or children $[9,10]$. Summing the time periods of intervention and follow-up revealed no significant impact of this SNP on weight changes after correcting for multiple testing according to a previous smaller study of our group [19]. In this study, another SNP in FTO (rs1558902, in high linkage disequilibrium; $\left.r^{2}=0.901, D^{\prime}=0.965\right)$ in intron 1 of FTO was used.

Interestingly, the SNP at the FTO was only related to weight regain after lifestyle intervention. This finding fits well to previous longitudinal population-based studies in 
children without interventions, which have demonstrated that the risk allele at the same SNP as analyzed in our study was associated with increased BMI z-scores at all points when starting at the age of 7 years until early adulthood [20, 21].

We can only speculate why this SNP is only related to weight regain but not to weight loss during intervention. Our finding supports the hypothesis that physical exercise, which is included as regular exercise in the lifestyle intervention 'Obeldicks', may counteract the obesity susceptibility allele(s) at FTO [3,4]. Of interest, lifestyle interventions with intensive physical exercise did not find a relationship between FTO polymorphism(s) and weight loss [8-10] in contrast to interventions without intensive physical exercise units $[7,11]$. A large-scale meta-analysis confirmed that the effect of FTO variation on the risk of obesity is significantly lower in physically active adults compared with sedentary people [22]. When regular physical exercise was very likely stopped at the end of intervention in most of our participants, the effect of FTO on BMI may be unmasked in the follow-up period probably explaining the relationship between the SNP at the FTO and weight regain. If the relationship between exercise behavior, the SNP at the FTO, and weight regain is true, this fits to the conclusion of several meta-analyses of long-term weight maintenance studies in adults and children that exercise is important to sustain weight loss $[23,24]$. Further longitudinal studies analyzing physical exercise during and after lifestyle intervention are necessary to prove this hypothesis.

In contrast to our findings, a large study in adults demonstrated no impact of SNP rs9939609 at FTO on weight regain [8]. One might speculate that the impact of this SNP on weight regain differs between adults and children besides other potential factors such as comorbidity and medication in adults influencing the relationship between FTO and weight regain. Indeed, the mentioned study of Delahanty et al. [8] was performed in adults with disturbances in glucose metabolism.

In concordance to previous studies $[9,25,26]$, we did not find an association of the risk allele at the rs9939609 FTO polymorphism to cardiovascular risk factors or their changes during lifestyle intervention when adjusting for BMI. Previous studies demonstrating an association between FTO obesity risk alleles and cardiovascular risk factors were not adjusted for BMI [26-28].

The strengths of our study are its longitudinal design and the follow-up period after the end of intervention. However, some limitations have to be kept in mind. First,
BMI percentiles were used to classify overweight. Although BMI is a good measure for overweight, one needs to be aware of its limitation as an indirect measure of fat mass. Second, due to the moderate sample size the findings have to be interpreted with caution. Third, we did not have a control group with obese children without intervention. However, previous longitudinal studies without intervention have demonstrated an increase of BMISDS in children carrying a risk allele at the FTO gene [2931]. Fourth, the SNP at the FTO does not allow estimating gene expression and the true genetic regulation connected to the different SNPs at the FTO gene are unknown. Finally, we are not able to differentiate the effect of diet and physical exercise on changes of weight status due to our study protocol. Especially, we have no objective measurements of physical activity after the end of intervention. However, it is unlikely that the participating children kept up with the same degree of exercise per week after the end of the intervention period. Furthermore, reliable measurements of dietary intake and physical exercise are very difficult to estimate due to underreporting or overstating, respectively. Future studies including objective measurements of physical activity (for example by accelerometer) should be performed to validate a potential impact of physical activity on the relationship between FTO and weight regain after end of a lifestyle intervention.

In summary, we described association of the obesity risk allele (A) at polymorphism rs9939609 in intron 1 of FTO on weight regain 1 year after a 1-year lifestyle intervention. Specifically tailored interventions for children with obesity susceptibility loci such as risk alleles in polymorphisms at FTO have to be developed, particularly when our findings have been confirmed in additional larger studies including objective measurements of physical exercise.

\section{Acknowledgements}

We thank the children for participating in this study. This work was supported by grants from the German Federal Ministry of Education and Research (BMBF): grant No. 01GS0820 of the National Genome Research Network, NGFN-Plus, and grant Nos. 01GI0839, 01 01GI1120A and 01 01GI1120B of the German Obesity Competence Network.

\section{Disclosure Statement}

The authors have no conflicts of interest to disclose. 


\section{References}

1 Speliotes EK, Willer CJ, Berndt SI, et al: Association analyses of 249,796 individuals reveal 18 new loci associated with body mass index. Nat Genet 2010;42:937-948.

$>2$ Vimaleswaran KS, Angquist L, Hansen RD, et al: Association between FTO variant and change in body weight and its interaction with dietary factors: the DiOGenes study. Obesity (Silver Spring) 2012;20:1669-1674.

-3 Perusse L, Rankinen T, Hagberg JM, et al: Advances in exercise, fitness, and performance genomics in 2012. Med Sci Sports Exerc 2013; 45:824-831.

$\checkmark 4$ Scott RA, Bailey ME, Moran CN, et al: FTO genotype and adiposity in children: physical activity levels influence the effect of the risk genotype in adolescent males. Eur J Hum Genet 2010;18:1339-1343.

5 Hakanen M, Raitakari OT, Lehtimaki T, et al: FTO genotype is associated with body mass index after the age of seven years but not with energy intake or leisure-time physical activity. J Clin Endocrinol Metab 2009;94:1281-1287.

6 Hubacek JA, Pikhart H, Peasey A, Kubinova $\mathrm{R}$, Bobak M: FTO variant, energy intake, physical activity and basal metabolic rate in Caucasians. The HAPIEE study. Physiol Res 2011;60:175-183

$>7$ Moleres A, Rendo-Urteaga T, Zulet MA, et al: Obesity susceptibility loci on body mass index and weight loss in Spanish adolescents after a lifestyle intervention. J Pediatr 2012;161:466470.

8 Delahanty LM, Pan Q, Jablonski KA, et al: Genetic predictors of weight loss and weight regain after intensive lifestyle modification, metformin treatment, or standard care in the Diabetes Prevention Program. Diabetes Care 2012;35:363-366.

$>9$ Schum J, Blumenstock G, Weber K, et al: Variants of the FTO gene in obese children and their impact on body composition and metabolism before and after lifestyle intervention. Exp Clin Endocrinol Diabetes 2012; 120:128-131.

$>10$ Muller TD, Hinney A, Scherag A, et al: 'Fat mass and obesity-associated' gene (FTO): no significant association of variant rs9939609 with weight loss in a lifestyle intervention and lipid metabolism markers in German obese children and adolescents. BMC Med Genet 2008;9:85.
11 Franks PW, Jablonski KA, Delahanty LM, et al: Assessing gene-treatment interactions at the FTO and INSIG2 loci on obesity-related traits in the Diabetes Prevention Program. Diabetologia 2008;51:2214-2223.

12 Tanofsky-Kraff M, Han JC, Anandalingam K, et al: The FTO gene rs9939609 obesity-risk allele and loss of control over eating. Am J Clin Nutr 2009;90:1483-1488.

13 Cole TJ, Bellizzi MC, Flegal KM, Dietz WH: Establishing a standard definition for child overweight and obesity worldwide: international survey. BMJ 2000;320:1240-1243.

14 Kromeyer-Hauschild K, Wabitsch M, Geller F, et al: Percentiles of body mass index in children and adolescents evaluated from different regional German studies. Monatsschr Kinderheilkd 2001;149:807-818.

15 National High Blood Pressure Education Program Working Group on High Blood Pressure in Children and Adolescents: The fourth report on the diagnosis, evaluation, and treat ment of high blood pressure in children and adolescents. Pediatrics 2004;114(suppl):555576.

16 Matthews DR, Hosker JP, Rudenski AS, Naylor BA, Treacher DF, Turner RC: Homeostasis model assessment: insulin resistance and $\beta$-cell function from fasting plasma glucose and insulin concentrations in man. Diabetologia 1985;28:412-419.

17 Reinehr T, Temmesfeld M, Kersting M, de Sousa G, Toschke AM: Four-year follow-up of children and adolescents participating in an obesity intervention program. Int J Obes (Lond) 2007;31:1074-1077.

18 Reinehr T: Lifestyle intervention in childhood obesity: changes and challenges. Nat Rev Endocrinol 2013;9:607-614.

19 Hinney A, Wolters B, Putter C, et al: No impact of obesity susceptibility loci on weigh regain after a lifestyle intervention in overweight children. J Pediatr Endocrinol Metab 2013;26:1209-1213.
20 Jess T, Zimmermann E, Kring SI, et al: Impact on weight dynamics and general growth of the common FTO rs9939609: a longitudinal Danish cohort study. Int J Obes (Lond) 2008;32: 1388-1394.

21 Elks CE, Loos RJ, Sharp SJ, et al: Genetic markers of adult obesity risk are associated with greater early infancy weight gain and growth. PLoS Med 2010; 7:e1000284.

22 Roth SM, Rankinen T, Hagberg JM, et al: Advances in exercise, fitness, and performance genomics in 2011. Med Sci Sports Exerc 2012; 44:809-817.

23 Weiss EC, Galuska DA, Kettel KL, Gillespie C, Serdula MK: Weight regain in US adults who experienced substantial weight loss, 19992002. Am J Prev Med 2007;33:34-40.

24 Wilfley DE, Stein RI, Saelens BE, et al: Efficacy of maintenance treatment approaches for childhood overweight: a randomized controlled trial. JAMA 2007;298:1661-1673.

25 Olza J, Ruperez AI, Gil-Campos M, et al: Influence of FTO variants on obesity, inflammation and cardiovascular disease risk biomarkers in Spanish children: a case-control multicentre study. BMC Med Genet 2013;14: 123.

26 Luczynski W, Zalewski G, Bossowski A: The association of the FTO rs9939609 polymorphism with obesity and metabolic risk factors for cardiovascular diseases in Polish children. J Physiol Pharmacol 2012;63:241-248.

27 Xi B, Zhao X, Shen Y, et al: Associations of obesity susceptibility loci with hypertension in Chinese children. Int J Obes (Lond) 2013; 37:926-930.

28 Zhao X, Xi B, Shen Y, et al: An obesity genetic risk score is associated with metabolic syndrome in Chinese children. Gene 2013;535: 299-302.

29 Warrington NM, Wu YY, Pennell CE, et al: Modelling BMI trajectories in children for genetic association studies. PLoS One 2013; 8:e53897.

>30 Lourenco BH, Qi L, Willett WC, Cardoso MA: FTO genotype, vitamin D status and weight gain during childhood. Diabetes 2013; 63:808-814.

-31 Hallman DM, Friedel VC, Eissa MA, et al: The association of variants in the FTO gene with longitudinal body mass index profiles in nonHispanic white children and adolescents. Int J Obes (Lond) 2012;36:61-68. 